\section{C4 \\ both increases \\ and decreases \\ in L6 \\ corticothalamic \\ activity can \\ disrupt RG \\ synapse \\ refinement}
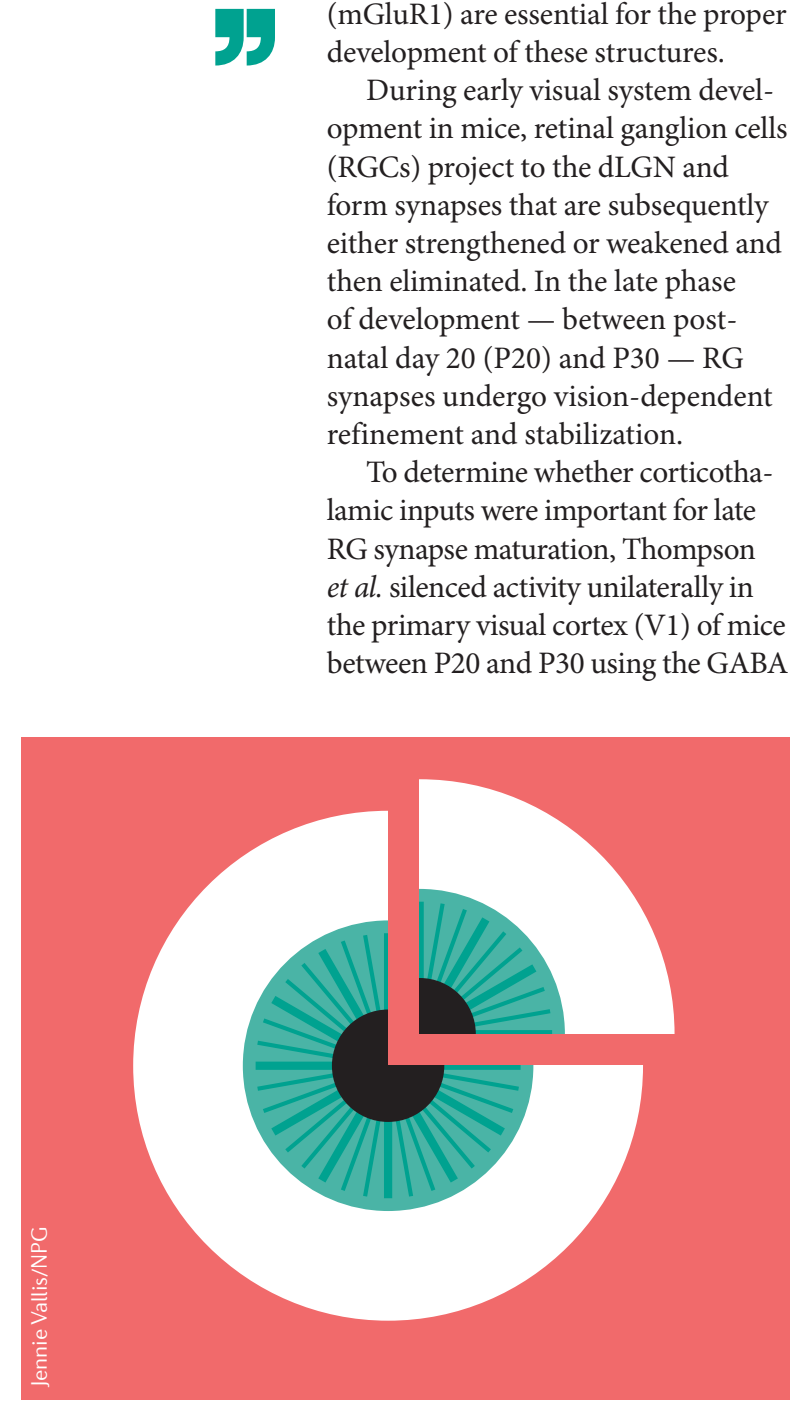

The development and maturation of the visual system - in particular, retinogeniculate $(R G)$ synapses in the dorsal lateral geniculate nucleus (dLGN) - rely on a series of intricate processes. Two recent studies further characterize the late phase of the development of RG synapses. Thompson et al. and Narushima et al. respectively show in mice that corticothalamic feedback and metabotropic glutamate receptor 1 (mGluR1) are essential for the proper development of these structures.

During early visual system development in mice, retinal ganglion cells (RGCs) project to the dLGN and form synapses that are subsequently either strengthened or weakened and then eliminated. In the late phase of development - between postnatal day 20 (P20) and P30 - RG synapses undergo vision-dependent refinement and stabilization.

To determine whether corticothalamic inputs were important for late RG synapse maturation, Thompson et al. silenced activity unilaterally in the primary visual cortex (V1) of mice between P20 and P30 using the GABA

NEURAL DEVELOPMENT

\title{
Sight development
}

type A receptor agonist muscimol, and then assessed RG synapse function in acute brain slices. The single-fibre fraction (SFF) - a measure of how much a single presynaptic fibre contributes to an excitatory postsynaptic current (EPSC) - of RG synapses in muscimol-treated mice was lower than in controls. Given that the SFF of a synapse is inversely correlated with the number of inputs to a postsynaptic neuron, this result suggests that the muscimol-treated mice have additional weak inputs to the thalamic neurons. Pharmacogenetic silencing of V1 layer 6 (L6) corticothalamic neurons between P20 and P30 had a similar effect on the RG SFF, confirming that these feedback neurons are necessary for proper RG refinement.

Interestingly, pharmacogenetically increasing L6 activity during this phase also led to reduced SFFs, suggesting that both increases and decreases in L6 corticothalamic activity can disrupt RG synapse refinement. Importantly, RG synapses are only vulnerable to pharmacogenetic inactivation of the L6 corticothalamic projections during the late phase: this manipulation had no effect on RG synapses if it was applied after P30. Thus, overall, this study shows that defects in cortical activity during this discrete late development phase can disrupt subcortical circuitry.

Narushima et al. asked whether mGluR1 plays a part in development. Immunohistochemistry revealed that mGluR1 is expressed in the dLGN during late development, and mGluR1-null mice showed normal early RG development. However, after P28, these animals showed reduced SFFs compared with wildtype controls. Therefore, mGluR1 is also important for RG synapse refinement.
Previous work showed that rearing mice in the dark during the late phase of visual development (late dark rearing) also leads to reduced SFFs at RG synapses, implying that RG synapse maturation is experience dependent. Here, Narushima et al. showed that late dark rearing does not affect the SFF in mGluR1-null mice, suggesting that mGluR1-mediated maturation and experience-dependent maturation share a common mechanism. Local pharmacological inhibition of dLGN mGluR1, or lentivirus-mediated knockdown of mGluR1 expression, led to reductions in the SFF. Moreover, targeted expression of mGluR1 in thalamic relay neurons of mGluR1-null mice at P14 rescued the SFF measured 2 weeks later. Finally, chronic pharmacological activation of mGluR1 during the late phase prevented reductions in the SFF resulting from late dark rearing. Together, these results suggest that mGluR1 is both necessary and sufficient for RG maturation.

These studies identify circuit-level and molecular mechanisms that are necessary for proper late development of the visual system. Intriguingly, Narushima et al. showed, using double-label immunoelectron microscopy, that mGluR1 is present at higher densities on the postsynaptic elements in corticogeniculate synapses, raising the possibility that the cortical feedback could act through mGluR1 on thalamic relay neurons, although this remains to be investigated.

Natasha Bray

ORIGINAL ARTICLES Thompson, A. D. et al Cortical feedback regulates feedforward retinogeniculate refinement. Neuron http://dx.doi. org/10.1016/j.neuron.2016.07.040 (2016) | Narushima, M. et al. The metabotropic glutamate receptor subtype 1 mediates experiencedependent maintenance of mature synaptic connectivity in the visual thalamus. Neuron http:// dx.doi.org/10.1016/j.neuron.2016.07.035 (2016) 